This item was submitted to Loughborough's Research Repository by the author.

Items in Figshare are protected by copyright, with all rights reserved, unless otherwise indicated.

\title{
Effect of long-term physical activity and acute exercise on markers of systemic inflammation in persons with chronic spinal cord injury: a systematic review
}

\section{PLEASE CITE THE PUBLISHED VERSION}

http://dx.doi.org/10.1016/j.apmr.2014.07.006

\section{PUBLISHER}

Elsevier / @ American Congress of Rehabilitation Medicine

\section{VERSION}

AM (Accepted Manuscript)

\section{PUBLISHER STATEMENT}

This work is made available according to the conditions of the Creative Commons Attribution-NonCommercialNoDerivatives 4.0 International (CC BY-NC-ND 4.0) licence. Full details of this licence are available at: https://creativecommons.org/licenses/by-nc-nd/4.0/

\section{LICENCE}

CC BY-NC-ND 4.0

\section{REPOSITORY RECORD}

Neefkes-Zonneveld, Celine R., Arjan J. Bakkum, Nicolette Bishop, Maurits W. van Tulder, and Thomas W. Janssen. 2019. "Effect of Long-term Physical Activity and Acute Exercise on Markers of Systemic Inflammation in Persons with Chronic Spinal Cord Injury: A Systematic Review". figshare.

https://hdl.handle.net/2134/16786. 
The Effect of Long-term Physical Activity and acute exercise on Markers of Systemic Inflammation in Persons with Chronic Spinal Cord Injury: A Systematic Review

ABSTRACT

Objectives: To evaluate the effect of long term physical activity (PA) and acute exercise on markers of systemic inflammation in persons with chronic spinal cord injury ( $\mathrm{SCl})$.

Data sources: We searched Pubmed (MEDline), Embase, CENTRAL, Cinahl and PEDro, involving variations of the MeSH headings: $\mathrm{SCl}, \mathrm{PA}$, exercise and inflammation,. No time or language restrictions were applied.

Study selection: Except for case reports, we included any type of study, both genders, all ages, with $\mathrm{SCl}$, resulting in 11 studies included. PA included leisure or work activity, including exercise.

Data extraction: Two authors independently scanned titles and abstracts, and read the articles included. One author extracted, while the second double-checked the data. The methodological quality and evidence were rated by the Cochrane Risk of Bias tool or the Newcastle-Ottawa Scale, and the GRADE approach.

Data synthesis: The included studies had a high risk of bias and 'very low' levels of evidence . Metaanalyses were performed (random effects model or generic inverse variance method). The acute interleukin 6 (IL-6) response to exercise was the same for $\mathrm{SCl}$ and able-bodied individuals $(\mathrm{p}=.91)$, however, responses were higher in paraplegia (PP) than in tetraplegia (TP),( weighted mean difference (WMD 1.19, $p<.00001$ and $0.25, p=0.003$, respectively). Compared to physically inactive people with $\mathrm{SCl}$, physically active people with $\mathrm{SCI}$ had lower plasma C-reactive protein (CRP) levels compared (WMD -0.38, $\mathrm{p}=.009$ ). CRP concentrations were lower post- than pre-exercise intervention (WMD -2.76, $\mathrm{p}=.0001)$.

Conclusions: PA and exercise may improve systemic markers of low-grade inflammation in $\mathrm{SCl}$, particularly IL-6 and CRP. The change in IL-6 and CRP is greater in PP compared to TP.

Keywords: inflammation markers; physical activity; spinal cord injury; paraplegia; tetraplegia. 


\section{Abbreviations}

BWSTT - body-weight-supported treadmill training

CRP - C-reactive protein

CVD - cardiovascular disease

FES - functional electrical stimulation

GRADE - Grading of Recommendations Assessment, Development and Evaluation IL - interleukin

IL-1ra - interleukin 1 receptor antagonist

LTPA - leisure time physical activity

MCP-1/CCL2 - monocyte chemotactic protein-1 or chemokine (C-C motif) ligand 2 NOS - Newcastle-Ottawa scale

PA - physical activity

PP - paraplegia

$\mathrm{SCl}$ - spinal cord injury

SMD - standard mean difference

SNS - sympathetic nervous system

TLR - Toll like receptor

TNF- $\alpha$ - tumour necrosis factor alpha

TP - tetraplegia

WMD - weighted mean difference 
2 Systemic low-grade inflammation, as expressed in 2-3 fold increases in levels of circulating

3 inflammatory markers, appears to be increased in persons with a spinal cord injury (SCl) compared with non-SCI $(1 ; 2)$. Chronic low-grade inflammation is a potential contributor to mortality and co-morbidity. Specific co-morbidities linked to elevated circulating inflammatory markers occur in considerable numbers of persons with $\mathrm{SCl}$, and include increased risks for cardiovascular disease (CVD) and respiratory disease, the two leading causes of death among persons with $\mathrm{SCl}(3 ; 4)$. In support of this, inflammatory cytokines are thought to play a role in pulmonary impairment, obesity and specifically metabolic syndrome, diabetes, some types of cancers, poor wound healing, indwelling urinary catheters and pressure ulcers (3).

Evidence in healthy able-bodied persons suggests that PA and exercise are related to a decreased risk of both developing and mortality from such chronic diseases by way of reducing levels of circulating markers of inflammation (5;6). Circulating levels of inflammatory markers are mediated by a variety of cytokines. These are immuno-modulating agents that can be classified as lymphokines, interleukins and chemokines, based on their function. Current evidence suggests that above a threshold intensity, contracting muscle releases myokines (cytokines released directly from working muscle) such as interleukin 6 (IL-6), resulting in large (>10 fold), short lasting increases in circulating IL-6 levels. This transient 'spike' in IL-6 levels appears to stimulate a counteractive release of anti-inflammatory cytokines, such as interleukin 1 receptor antagonist (IL-1ra), thus creating a circulating antiinflammatory environment with each bout of exercise $(5 ; 16 ; 17)$. IL-6 release from muscle is also associated with several positive metabolic effects including enhanced lipolysis and improved insulin sensitivity. Interleukin 15 (IL-15), another key inflammatory myokine released from the working muscles, seems to be involved in increasing an anti-inflammatory environment. IL-15 possesses anabolic effects on skeletal muscle and plays a role in reducing adipose tissue mass, thereby influencing muscle-fat crosstalk (7). 
In addition to these acute exercise effects, regular PA is also associated with higher circulating numbers of regulatory $\mathrm{T}$ cells that release the anti-inflammatory cytokine IL-10 (5). Furthermore, regular PA appears to both reduce the infiltration of inflammatory immune cells into adipose tissue and stimulate phenotypic alterations of monocytes within adipose tissue, with cells switching to an anti-inflammatory phenotype. These events, along with an exerciseinduced down-regulation of monocyte toll-like receptor expression leading to reduced monocyte activation $(8 ; 9)$, are associated with reduced release of pro-inflammatory adipokines (cytokines release from adipose tissue) such as tumor necrosis factor- $\alpha$ (TNF- $\alpha$ ), monocyte chemotactic protein-1 (MCP-1/CCL2) and IL-6(5;7). Importantly, this reduced long-lasting circulating IL-6 response (as opposed to the short, sharp large increases associated with muscle contraction) also reduces the stimulus for the liver to release CRP.

Taken together, it is not surprising that exercise is considered best practice to enhance health in both healthy people and people with chronic disease (10). However, persons with $\mathrm{SCl}$ are amongst the most sedentary and inactive people worldwide (11) as a consequence of loss of function and enforced behavior. $\mathrm{SCl}$ is heterogeneous by nature and can either be characterized by incomplete or complete tetraplegia (C1-C8) or paraplegia (PP) (T1 and below). Persons with the same level of $\mathrm{SCl}$ can differ in symptom display and abilities, partially caused by the degree of sympathetic nervous system (SNS) dysfunction and the quantity of muscle mass that can be activated (12). Given the role of active muscle in the anti-inflammatory effects of exercise, the decreased muscle mass and impaired muscle innervation and function in people with $\mathrm{SCl}$ is expected to limit potential anti-inflammatory benefits $(12 ; 13)$. Furthermore, in able-bodied populations CRP is reported to be lower in response to regular $\mathrm{PA}$ and linked with $\mathrm{BMI}$ as a risk factor for developing CVD $(7 ; 8)$.

\section{Thus far, the effects of PA and exercise have been investigated more extensively in healthy} able-bodied persons, though the effects in persons with $\mathrm{SCl}$ are not well known. Therefore, the aim of this systematic review was to evaluate the effect of long-term PA and acute exercise on markers of systemic inflammation in persons with chronic SCl. In this systematic 
review, high versus low PA levels, different exercise modalities, and different levels of SCI were evaluated, and a comparison between persons with and without SCI was made.

60

\section{Methods}

\section{Inclusion criteria}

Any type of study was included, except for case reports, with both male and female participants of all ages with either acute or chronic ( $\geq 1$ year post injury) PP or TP. PA consisted of leisure or work activity, including exercise.

\section{Comparisons}

In the review protocol we determined the following a priori comparisons of effect to investigate the acute- and long-term response on levels of inflammatory markers in SCl:

- Exercise vs. no exercise;

- Low PA vs. high PA levels;

- $\quad$ Aerobic vs. strengthening exercises;

- Aerobic and strengthening exercise vs. aerobic or strengthening or no exercise;

- Exercise in acute SCI versus chronic SCI;

- Exercise in SCl vs. exercise in able-bodied persons.

\section{Outcome measures}

The outcome measures assessed for the acute effects of exercise were IL-6, IL-1ra and IL10 (14-16). The long-term effect key inflammatory markers studied were CRP, TNF- $\alpha$ and MCP-1/CCL2 (5;6;17).

Search strategy

The search strategy was developed in close collaboration with a medical information specialist, and the final version was approved by two assessors. The databases used were: Pubmed (MEDline), Embase, Cochrane Central Register of Controlled Trials (CENTRAL), 
Cinahl and PEDro, including articles up to March $19^{\text {th }}, 2013$. No time or language restrictions were applied and the strategy included MeSH headings and keyword searches involving variations of the following principle terms: spinal cord injuries, physical activity, exercise, wheelchair sports, electrical stimulation, inflammation, cytokines, myokines and adipokines. The search was complemented by scanning reference lists of the selected publications. Some authors were contacted for extra data information.

\section{Data collection and analysis}

The two review assessors independently scanned the titles and abstracts before reaching consensus regarding the articles needed to be included. In case of disagreements, a third reviewer was involved. The electronic references were documented using Reference Manager 12.03 bibliographic software. One of the assessors extracted relevant data from the included articles. The data extraction was checked by a second assessor and discussed within the group of authors before analysis took place.

\section{Assessment of risk of bias and level of evidence}

The two assessors assessed the risk of bias of the included articles by using the Cochrane Risk of Bias tool in case of prospective controlled trials, and the Newcastle-Ottawa Scale (NOS) in case of observational studies (18). Because of its validation, the NOS checklist for cohorts was used to assess the included cross-sectional studies. Case series were considered having a high risk of bias. In addition the two assessors evaluated the overall strength of evidence by using the Grading of Recommendations Assessment, Development and Evaluation (GRADE) approach (19). GRADE identifies risk of bias, imprecision, inconsistency, indirectness and publication bias, thereby focusing on each important outcome across the included studies (19). GRADE specifies four categories of quality (i.e. high, moderate, low and very low) that are applied to the total body of evidence. The final rating of the overall evidence of quality (performed with GRADEprofiler version 3.6) includes the validity, precision, consistency, and applicability of the estimates (19). 
Meta-analysis

All statistical analyses were performed using Review Manager Version 5.2. When possible, a meta-analysis was performed. Study data were tested on heterogeneity by the eye-ball test (evaluating overlapping confidence intervals), applying a test for homogeneity (Q), and by quantifying the heterogeneity $\left(\mathrm{I}^{2}\right)$. Because some variation among studies was expected, a random-effects model was used. For continuous outcomes being measured with identical scale, the weighted mean difference (WMD) was used as effect estimate; for studies with different scales, the standardized mean difference (SMD) was used. For studies with a pre and post measurement, the results were pooled with a generic inverse variance method, using the average difference and standard error per group.

\section{Results}

\section{Search strategy}

A total of 2037 articles were retrieved from the search process, of which 1825 articles remained after removing duplicates. The assessment of the titles and abstracts resulted in 13 potential articles, of which the full articles were obtained. After reading the full articles, 11 studies were included in this review (20-30). A summary of the search process is presented in Figure 1. No randomized-controlled trials were identified. However, three case series (35$37)$, five cross-sectional studies $(29 ; 33 ; 34 ; 38 ; 39)$ and three prospective (non-randomized) controlled trials (30-32) were disclosed. The study characteristics are included in Table 1. The included 11 studies involved 328 participants in total, of which only 15 were female. The age ranged from 22 to 70 years and the time since injury ranged from 2 to 39 years. Three studies included females $(26 ; 28 ; 29)$ and two studies included persons with PP and TP in separate groups (21;29). Participants were recruited from medical records, (rehabilitation) hospitals and clinics and by active recruitment in the United States, Canada, Brazil, Japan, Great Britain and Italy.

\section{Comparisons and interventions}


145

146

147

148

149

150

151

152

153

154

155

156

157

158

159

160

161

162

163

164

165

166

167

168

169

170

171

172

173

Within the acute response comparison 'Exercise in persons with $\mathrm{SCl}$ versus exercise in non-

$\mathrm{SCl}$ (other wheelchair users) or able-bodied persons', the exercise interventions varied

widely. (Table 1). In all three included prospective controlled trials, one exercise session was applied, comprising of arm cranking ergometer exercise of different duration (31; 32), or submaximal or graded exercise wheelchair testing on a motorized treadmill (21).

The effect of 'pre to post aerobic exercise training' was compared in all of the case series. Two of the three case series investigated the long-term response to aerobic exercise. One of these studies applied functional electrical stimulation (FES cycling (26), while the other applied body-weight-supported treadmill training (BWSTT) with gradually reduced support as tolerated (28). In the last case series, the acute response of a competition wheelchair basketball match was investigated (27).

Within different cut-off points or parameters, the long-term comparison 'low PA versus high PA in SCl' was explored in the cross-sectional studies. One of the five cross-sectional studies (Table 1), compared participants with low leisure time physical activity (LTPA) $(<25$ $\min /$ day) to participants with high LTPA ( $\geq 25 \mathrm{~min} /$ day); analyses were performed for the whole group and separately for the TP and PP groups (29). Another cross-sectional study compared those who participated in PA for a total of 150 min/week with non-physically active participants (33). Yet another study compared tertiles of PA in metabolic equivalents (METs) hours per day (29). Furthermore, one study analyzed associations between peak oxygen uptake ( $\mathrm{VO}_{2 \text { peak; }}$; absolute and relative), PA and CRP (30), while the last study compared CRP in mobility mode (motorized wheelchair, manual wheelchair, walks with an aid and walks without an aid) (25).

\section{Outcome measures}

The outcome measures (Table 1) of the three prospective controlled trials included IL-6 (22;23), IL-10, IL-1ra (21) and TNF- $\alpha$ levels (21;23). Lastly, it included CRP (23). The case series used IL-6, TNF- $\alpha$ and CRP as outcome (26-28). In the cross-sectional studies CRP 
$174(20 ; 30)$ and IL-6 (30) were used as outcome measure in correlation with PA, while the last

175 study used the outcome of CRP in association with locomotive mode (25).

176

177

178

179

180

181

182

183

184

185

186

187

188

189

190

191

192

193

194

195

196

197

198

199

200

201

202

Risk of bias

Prospective controlled trials

The risk of bias assessment of the prospective controlled trials is summarized in Table 2.

In all three trials the risk of selection bias was considered high because the studies were not randomized. Since the blood analyses of all three studies were performed in a laboratory setting and in two of the studies $(31 ; 32)$ duplicate blood samples were taken, the risk of performance bias was judged as low. All three trials had unclear risk of attrition bias $(30 ; 36)$. The risk of selective reporting bias was judged low, because the study protocols of all three studies were available and all included outcomes were reported. An additional risk of indirectness was considered to be present, because by selecting men only and in one case these being wheelchair athletes, the study populations were not true representatives of the whole SCI population.

\section{Cross-sectional studies}

The risk of bias assessed is summarized in Table 3. Except for the Buchholz study (29), the risk of selection bias was judged high as a result of selecting men with $\mathrm{SCl}$ only, the studies being cross-sectional, and the self-reported PA in four out of five studies. However, the selection of the non-exposed was drawn from the same cohort in all five studies attenuating selection bias somewhat. The risk of attrition bias was judged low in four of the five studies $(20 ; 25 ; 29 ; 30)$, in which was controlled for at least one or more key factors. Since in all five studies the blood analyses were done in a laboratory, the detection bias was judged low. The time of follow-up was lacking since all five studies had a cross-sectional design and causal conclusions cannot be drawn upon the results.

\section{Case series}


203

204

205

206

207

208

209

210

211

212

213

214

215

216

217

218

219

220

221

222

223

224

225

226

227

228

229

230

231

The three included case series were not formally assessed, however, it was noticed that two of these studies selected a population that was representative of the adult SCI population $(26 ; 28)$.

\section{Effects of interventions}

The summary of findings for the main comparisons (Table 4) shows the results of the overall quality of evidence. The evidence was rated 'very low' for the 'acute effect of exercise on the IL-6 response compared to pre-exercise in SCI versus able-bodied participants', the 'longterm effect on CRP between PA and non-PA in SCl' and for the long-term effect of PA on CRP level in SCl.

\section{Systemic inflammatory responses to acute exercise}

Exercise in persons with SCI versus exercise in non-SCl or able-bodied persons

Baseline IL-6 was significantly higher in persons with chronic SCl, $(2.18 \pm 0.44 \mathrm{pg} / \mathrm{ml})$ than in able-bodied participants in one study $(1.02 \pm 0.22 \mathrm{pg} / \mathrm{ml})(\mathrm{p}<0.05)(22)$. However, Umemoto et al. (23) reported no differences in plasma IL-6 reaction between the SCI and able-bodied group, while detecting significant increases in circulating IL-6 at baseline and before exercise in $\mathrm{SCl}$ compared to able-bodied persons, and during, immediately after and 2 hours after exercise for both groups. In addition, they reported higher CRP values in the SCI group compared with the able-bodied group throughout the study, while the CRP and TNF- $\alpha$ did not change in either group throughout the study (23). The third study reported a five-fold elevation of circulatory IL-6 compared with pre-exercise in PP and Non-SCl groups. Both groups showed a significant ( $p=.003$ for interaction) effect directly post exercise and 30 minutes after exercise. No significant circulatory IL-6 changes were detected in the TP group. There was no effect on plasma IL-10 concentration for any groups in response to exercise, however, baseline levels of IL-10 were higher in the TP and PP groups compared with the non-SCI group ( $p=.001$ for group). In addition, no significant interaction effects or main effects of group or time for plasma concentrations of IL-1ra and TNF- $\alpha$ were found (21). All 
232 three studies included only adult males. When the results of the 3 studies were pooled for

233 analysis comparing the $\mathrm{SCl}$ groups with able-bodied participants (Figure 2), there was no

234 effect of exercise on plasma IL-6 concentrations $(p=0.91)$.

236 Exercise in SCl only

237 We did not define this subgroup a priori, however, due to substantial heterogeneity we

238 looked for a trend to see if this would support other findings of this review. There was only

239 one study that evaluated the acute effect of exercise on inflammation in 5 athletes with SCI

240 (T7 - T12) with no control group. The athletes engaged in a competition wheelchair

241 basketball game. The IL-6 levels changed from $1.11 \pm 0.66$ pre-game to $2.5 \pm 1.29 \mathrm{pg} / \mathrm{ml}$ post-

242 game $(p<.05)(27)$. In addition, we were able to retrieve two more PP groups to add and

243 perform a subgroup analysis (not shown). The WMD was $1.19 \mathrm{pg} / \mathrm{ml}$, with a 95\% Cl of 1.11

244 to $1.28(p<0.001)$, with no heterogeneity, indicating an increase of IL-6 post exercise

245 compared to pre-exercise in PP only.

247 We were also able to retrieve two TP groups with a pre- and post exercise comparison (not

248 shown). The pooled WMD was $0.25 \mathrm{pg} / \mathrm{ml}$, with a $95 \% \mathrm{Cl}$ of 0.09 to $0.42(p=0.003)$, while

249 the heterogeneity was negligible $\left(I^{2}=14 \%\right)$. However, conclusions should be carefully

250 drawn, because of the post-hoc subgroup analysis, the effect measure being estimated from

251 a figure, the imputed SD of one study (22), and the small sample size.

252

253 We did not identify any studies evaluating the following acute response comparisons:

254 Exercise in SCl vs. no exercise in SCl; Aerobic exercise versus strengthening exercise in

$255 \mathrm{SCl}$; Aerobic- and strengthening exercise versus aerobic or strengthening exercise in $\mathrm{SCl}$;

256 Exercise in acute $\mathrm{SCl}$ versus chronic SCl. 
261 Four cross-sectional studies reported outcomes for this comparison (29;33;38;39). The effect

262 of PA on circulatory CRP (3 studies, $N=47$ ) had a WMD of $-0.38 \mathrm{mg} / \mathrm{L} ; \mathrm{Cl}$ of -0.67 to -0.09

$263(p=0.009)$ indicating an inverse association of PA with CRP (Figure 3).

264 When we investigated the effect of adding mode of mobility data from Morse et al.

265 (34) to the association between PA and circulatory CRP in SCI (Figure 4), the effect was

266 attenuated and had a WMD of $-0.53 \mathrm{mg} / \mathrm{L} ; 95 \% \mathrm{Cl}-1.04$ to $-0.03(p=0.04)$. The heterogeneity

267 can be explained by the difference between mode of mobility and non-PA versus PA.

268

269 Physical activity in tetraplegia versus paraplegia

270 The studies did not allow a comparison of PA in TP and PP. Although, two studies $(24 ; 29)$

271 showed no association, as a result between PA and circulatory CRP level for TP (Figure 5),

272 the WMD was $-0.11 \mathrm{mg} / \mathrm{L} ; 95 \% \mathrm{Cl}$ of -0.63 to $0.41 ; \mathrm{p}=0.68$; and $\mathrm{I}^{2}=6 \%$.

273

274

275

276

277

278

279

280

281

282

283

284

285

286

287

288

289

\section{Effect of regular exercise in SCI}

Exercise in SCl only

We did not define this subgroup a priori, however, we identified two studies evaluating the longitudinal effects of exercise in participants with $\mathrm{SCl}$ only without a control group. Both studies were similar in gender distribution equal to the general $\mathrm{SCl}$ population. One study resulted in significant decreases of base levels of CRP, IL- 6 and TNF- $\alpha$, after 2 to 3 times per week of FES cycling for 10 weeks $(p<.05)(26)$. The other study resulted in a mean reduction in CRP of -1.54 (0.187), $p=0.0022$ (signed rank one-tailed test) after 5 times per week, 45 minutes per day for 6 weeks of BWSTT (28). Both results would indicate that the combinations of duration, frequency, intensity and type of exercise of these interventions are sufficient to elicit reduced base CRP levels in persons with $\mathrm{SCl}$. When we pooled both CRP effects (Figure 6), it resulted in a WMD of $-2.76 ; 95 \% \mathrm{Cl}-4.19$ to $-1.34(p=0.0001)$, suggesting an inverse relationship between long-term exercise, either FES or BWSTT, and

CRP in SCl. 
We did not identify any studies evaluating the following long-term comparisons: Acute versus

chronic SCl; Physical activity in SCl versus able-bodied participants; Aerobic exercise versus strengthening exercise in $\mathrm{SCl}$; Aerobic exercise and strengthening exercise versus aerobic or strengthening exercise in $\mathrm{SCl}$.

\section{Adverse events}

No adverse events were indicated.

\section{Discussion}

301 The response of circulating IL-6 to acute exercise was not different between persons with $302 \mathrm{SCl}$ compared with non-SCl or able-bodied persons. Subgroup analyses showed significantly 303 higher plasma IL-6 levels for TP in response to one bout of exercise, however, these 304 increases were smaller than those in persons with PP. This indicates that plasma IL-6 increases in response to acute exercise in both able-bodied and persons with $\mathrm{SCl}$.

The results from studies of regular PA demonstrate that high levels of regular PA are associated with lower resting levels of circulating CRP compared with low PA in SCl. However, when the same association was tested cross-sectionally in persons with TP, no significant effect could be established. The association between PA and a low resting circulating CRP concentrations was supported by the regular exercise interventions in $\mathrm{SCl}$,

312 however, the results appear to be largely attributable to those with PP (PP groups N=18, 313 combined TP and PP group $\mathrm{N}=18$ ).

The strengths, to our knowledge, are that this systematic review is the first that included a meta-analysis on the effect of PA on the inflammatory response in $\mathrm{SCl}$, and the first that investigated both long-term- and acute effects of PA in SCI. In addition, we identified the gap in $\mathrm{SCl}$ research. Indicating, first that there is no knowledge on the effect of strength exercise 
319 in $\mathrm{SCl}$, and second, there is no strong evidence for the short- or long-term effect of both

320 cardio- and strength training in different SCI populations.

322 Four published reviews, addressing cardiovascular and metabolic diseases and PA in SCI, 323 also discussed PA and systemic inflammation (31-34). None of these reviews reported a 324 search strategy or performed meta-analyses. They included three observational studies of 325 the eleven studies $(25 ; 29 ; 30)$ that were included in the current review. In agreement with 326 earlier studies $(1 ; 2 ; 4)$, we found indications of elevated resting levels of plasma CRP and IL3276 in persons with $\mathrm{SCl}$, while also exhibiting elevations in response to exercise. However, the 328 magnitude of the response was dependent on duration, intensity and type of exercise as

329 seen in the separate interventions. Diversity in type of exercise or level of PA was also 330 observed in our review and might explain the statistical heterogeneity. Further heterogeneity 331 can be explained by the population differences of the included studies. The SCI group 332 consisted of males with lesions at C6 - C7 in one study (22), and of males with lesion at T6 333 T10, while the third study included both a TP group $(\mathrm{C} 6-\mathrm{C} 7)$ and a PP group (T10 - L6)

334 (22). Third, in the first two studies the controls were able-bodied (22;23), while the last study 335 included non-SCl elite wheelchair athletes as controls (21). The overall heterogeneity 336 between the studies hampers a clear investigation of an acute dose-response relationship in 337 any type of exercise between and CRP in PP and TP as seen in non-SCl, independent from 338 baseline levels $(17 ; 35-39)$.

340 Inflammation markers are elevated in SCl compared to non-SCl, and similar to our findings, 341 Gibson et al. (1) demonstrated that CRP was clinically high in persons with SCI, which 342 according to the American Heart Association (AHA) is associated with a high risk of CVD.

343 Moreover, they concluded that CRP was elevated in PP and even more so in TP, implicating 344 a different inflammatory response between PP and TP(1). When the long-term effects were 345 pooled, we found no significant difference in CRP level between PA and non-PA in TP, in 346 contrast to the significant whole SCI group effect. However, the response of IL-6 to acute exercise in TP indicated a significant effect in the meta-analysis, and contradicting effects 
among the studies, while the IL-6 response to acute exercise in PP was both significant in

349 the meta-analysis and in the studies. The difference can be explained, first by a possible underpowered analysis by way of low numbers of TP, or second of a likely larger active

351 muscle mass, and lastly by a consequent larger voluntary muscle contraction, allowing

352 persons with PP to elicit more myokines from the working muscle compared to persons with

353 TP. (40;41). However, it does not explain our significant finding of the pooled response of

354 elevated IL-6 in response to acute exercise in those with TP, and further investigation from 355 large, well controlled studies is necessary to clarify.

The studies included in his review were not sufficiently powered. However, expectations of increasing levels of inflammatory markers as an acute response to exercise, like in ablebodied persons, and decreasing base levels of inflammatory markers as a long-term response, both in comparison to pre-exercise levels were confirmed in meta-analyses for IL6 and CRP respectively. Furthermore, there is some support that exercise performed at least at $60 \%$ of $\mathrm{VO}_{2}$ peak, with a duration of 2 hours, or graded exercise until exhaustion, are both sufficient to elicit a significant increase of IL-6 above pre-exercise levels in persons with a $\mathrm{SCl}(26 ; 28)$. When performed three to five times per week for 6 to 10 consecutive weeks, the resting level of CRP will decrease significantly, therefore potentially reducing the risk of CVD and respiratory disease in persons with $\mathrm{SCl}$. However, the external validity of the studies included in this review may be low, on account of the inclusion of few women. Although the influence of gender on the systemic inflammatory response to PA in SCl has not yet been investigated, it is known that there are sex differences in IL-6 responses both at rest and in response to exercise. At rest the difference may be enhanced by females taking oral

371 contraceptives, while the exercise-induced II-6 response in females is prolonged after

372 exercise when the male level is already decreasing $(42 ; 43)$.

374 For clinical implication, ,the sub-group analysis of level and severity of injury and the time since injury should be investigated.. To indicate if and from what timepoint since injury exercise is beneficial for which type of SCI. In addition, information regarding the occurrence 
377 of adverse effects (if any) should be reported, considering arm- and shoulder injuries are

378 very common in SCI. Furthermore, the effect of PA on circulating inflammatory markers in PP

379 and TP should be investigated in more detail to add statistical power, insight and overall

380 knowledge and build up evidence on the effect of exercise in SCl. This would include, a

381 possible dose-response relationship between the type, duration, frequency and intensity of

382 PA and lower levels circulating inflammatory markers of chronic low-grade inflammation.

383 Knowledge about possible dose-response relationships, for the different types of SCI to start

384 at a specific time since injury, will aid the therapeutic process.

385

386 Even though this study may have assessed some relevant factors, the estimate of effect

387 remains uncertain with a need for more valid answers through research. Heterogeneity, the

388 small number of studies, the small study populations and selection bias led to a GRADE

389 quality score of 'very low' for all comparisons. Therefore, future studies should include a

390 control group, a larger number of participants, more women, and various levels of SCl.

391 However, recruiting larger sample sizes in $\mathrm{SCl}$ may prove difficult considering that $\mathrm{SCl}$ is a

392 rare disorder and heterogeneous by nature. It seems unethical to withhold treatment for the

393 control group when exercise facilities are difficult to attain or to reach, while in addition, it is

394 many persons with SCl find it difficult to overcome barriers to begin exercising (11). Given

395 these difficulties, it may be plausible to develop a methodological assessment tool. A new

396 tool for non-double blinded randomized trials, in contrast to the existing tools, should weigh

397 the biological implications of the outcomes that can be of relative importance over the

398 methodological quality for studies that explore interventions that cannot be fully blinded by

399 definition. Non blinded studies such as exercise or food related interventions, and/or in rare

400 disorders (small sample sizes). The tool may account for blinded result assessment by the

401 statistician, in conjunction with the weighed biological significance, thereby adding to the

402 power of the body of evidence.

403

404 Some limitations of this review are, the use of the NOS scale for cohort studies to assess 


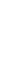

assessment of these studies on all items regarding longitudinal aspects. In addition, we did not identify negative studies, possibly enhancing publication bias and overestimation of the results. One last important limitation to applicability of the evidence is that PA had different cut-off points in different studies and exercise was diverse in type, duration and intensity. Consequently, strong evidence is lacking on a possible dose-response association of PA and inflammatory markers in SCl.

\section{Conclusions}

The findings of the current study suggest a significant increase in circulating IL-6 concentrations directly after moderate to vigorous exercise for persons with SCl. The effects of long-term exercise suggest a significant association and effect between PA and a reduction of circulating CRP, and some indication of II-6 and TNF- $\alpha$ plasma reduction in $\mathrm{SCI}$, while resting levels of IL-6, CRP and IL-10 in SCI were high compared to able-bodied persons. The exercise response appears to be more pronounced in persons with $\mathrm{PP}$, with conflicting results for persons with TP. In addition, there does not seem to be a difference in the response of circulating inflammatory markers to exercise between persons with $\mathrm{SCl}$ and able-bodied persons, another indication that PA and exercise may be also beneficial for $\mathrm{SCl}$. However, the quality of evidence supporting a reduced risk of pulmonary disease and CVD in $\mathrm{SCl}$ via reductions in chronic systemic inflammatory markers with exercise is very low. Further research of higher methodological quality is needed. 
(1) Gibson AE, Buchholz AC, Martin Ginis KA. C-Reactive protein in adults with chronic spinal cord injury: increased chronic inflammation in tetraplegia vs paraplegia. Spinal Cord 2008 Sep;46(9):616-21.

(2) Davies AL, Hayes KC, Dekaban GA. Clinical correlates of elevated serum concentrations of cytokines and autoantibodies in patients with spinal cord injury. Arch Phys Med Rehabil 2007 Nov;88(11):1384-93.

(3) DeVivo MJ, rause JS, Lammertse DP. Recent trends in mortality and causes of death among persons with spinal cord injury. Arch Phys Med Rehabil 1999 Nov;80(11):1411-9 1999 Nov 8;80(11):1411-9.

448

449

450

451

452

453

454

455

456

457

458

459

460

461

462

463

464

465

466

467

468

469

470

471

472

473

474

475
(4) Wang TD, Wang YH, Huang TS, Su TC, Pan SL, Chen SY. Circulating levels of markers of inflammation and endothelial activation are increased in men with chronic spinal cord injury. J Formos Med Assoc 2007 Nov;106(11):919-28.

(5) Gleeson M, Bishop NC, Stensel DJ, Lindley MR, Mastana SS, Nimmo MA. The antiinflammatory effects of exercise: mechanisms and implications for the prevention and treatment of disease. Nat Rev Immunol 2011 Sep;11(9):607-15.

(6) Beavers KM, Brinkley TE, Nicklas BJ. Effect of exercise training on chronic inflammation. Clin Chim Acta 2010 Jun 3;411(11-12):785-93.

(7) Pedersen BK. Exercise-induced myokines and their role in chronic diseases. Brain Behav Immun 2011 Jul;25(5):811-6.

(8) Oh EG, Bang SY, Kim SH, Hyun SS, Chu SH, Jeon JY, et al. Therapeutic lifestyle modification program reduces plasma levels of the chemokines CRP and MCP-1 in subjects with metabolic syndrome. Biol Res Nurs 2013 Jan;15(1):48-55.

(9) Troseid M, Lappegard KT, Claudi T, Damas JK, Morkrid L, Brendberg R, et al. Exercise reduces plasma levels of the chemokines MCP-1 and IL-8 in subjects with the metabolic syndrome. Eur Heart J 2004 Feb;25(4):349-55.

(10) Garber CE, Blissmer B, Deschenes MR, Franklin BA, Lamonte MJ, Lee IM, et al. American College of Sports Medicine position stand. Quantity and quality of exercise for developing and maintaining cardiorespiratory, musculoskeletal, and neuromotor fitness in apparently healthy adults: guidance for prescribing exercise. Med Sci Sports Exerc 2011 Jul;43(7):1334-59.

(11) Martin Ginis KA, Hicks LA. Exercise research issues in the spinal cord injured population. Exerc Sport Sci Rev 2005 Jan 1;33(1):49-53.

(12) Teasell RW, Arnold JM, Krassioukov A, Delaney GA. Cardiovascular consequences of loss of supraspinal control of the sympathetic nervous system after spinal cord injury. Arch Phys Med Rehabil 2000 Apr;81(4):506-16.

(13) Petersen AM, Pedersen BK. The anti-inflammatory effect of exercise. J Appl Physiol 2005 Apr;98(4):1154-62. 
(14) Chatzinikolaou A, Fatouros IG, Gourgoulis V, Avloniti A, Jamurtas AZ, Nikolaidis MG, et al. Time course of changes in performance and inflammatory responses after acute plyometric exercise. J Strength Cond Res 2010 May;24(5):1389-98.

(15) Pedersen BK, Hoffman-Goetz L. Exercise and the immune system: regulation, integration, and adaptation. Physiol Rev 2000 Jul;80(3):1055-81.

(16) Pedersen BK. Edward F. Adolph distinguished lecture: muscle as an endocrine organ: IL-6 and other myokines. J Appl Physiol 2009 Oct;107(4):1006-14.

(17) Geffken DF, Cushman M, Burke GL, Polak JF, Sakkinen PA, Tracy RP. Association between physical activity and markers of inflammation in a healthy elderly population. Am J Epidemiol 2001 Feb 1;153(3):242-50.

(18) Wells GA, Shea B, Peterson J, Losos M, Tugwell P. The Newcastle-Ottawa Scale (NOS) for assessing the quality of nonrandomised studies in meta-analyses. http://www.ohri.ca/programs/clinical epidemiology/oxford.asp . 2013.

Ref Type: Online Source

(19) Balshem $H$, Helfand M, Schunemann HJ, Oxman AD, Kunz R, Brozek J, et al. GRADE guidelines: 3. Rating the quality of evidence. J Clin Epidemiol 2011 Apr;64(4):401-6.

(20) Liang H, Mojtahedi MC, Chen D, Braunschweig CL. Elevated C-reactive protein associated with decreased high-density lipoprotein cholesterol in men with spinal cord injury. Arch Phys Med Rehabil 2008 Jan;89(1):36-41.

(21) Paulson TA, Goosey-Tolfrey VL, Lenton JP, Leicht CA, Bishop NC. Spinal Cord Injury Level and the Circulating Cytokine Response to Strenuous Exercise. Med Sci Sports Exerc 2013 Mar 7.

(22) Kouda K, Furusawa K, Sugiyama H, Sumiya T, Ito T, Tajima F, et al. Does 20-min arm crank ergometer exercise increase plasma interleukin- 6 in individuals with cervical spinal cord injury? Eur J Appl Physiol 2012 Feb;112(2):597-604.

(23) Umemoto Y, Furusawa K, Kouda K, Sasaki Y, Kanno N, Kojima D, et al. Plasma IL6 levels during arm exercise in persons with spinal cord injury. Spinal Cord 2011 Dec;49(12):1182-7.

(24) Koury JC, Passos MC, Figueiredo FA, Chain A, Franco JG. Time of physical exercise practice after injury in cervical spinal cord-injured men is related to the increase in insulin sensitivity. Spinal Cord 2013 Feb;51(2):116-9.

(25) Morse LR, Stolzmann K, Nguyen HP, Jain NB, Zayac C, Gagnon DR, et al. Association Between Mobility Mode and C-Reactive Protein Levels in Men With Chronic Spinal Cord Injury. Archives of Physical Medicine and Rehabilitation 2008 Apr;89(4):726-31.

(26) Griffin L, Decker MJ, Hwang JY, Wang B, Kitchen K, Ding Z, et al. Functional electrical stimulation cycling improves body composition, metabolic and neural factors in persons with spinal cord injury. J Electromyogr Kinesiol 2009 Aug;19(4):614-22.

(27) Kinoshita T, Nakamura T, Umemoto Y, Kojima D, Moriki T, Mitsui T, et al. Increase in interleukin-6 immediately after wheelchair basketball games in persons with spinal cord injury: preliminary report. Spinal Cord 2013. 
(28) Turiel M, Sitia S, Cicala S, Magagnin V, Bo I, Porta A, et al. Robotic treadmill training improves cardiovascular function in spinal cord injury patients. International Journal of Cardiology 2011;149(3):323-9.

(29) Buchholz AC, Martin Ginis KA, Bray SR, Craven BC, Hicks AL, Hayes KC, et al. Greater daily leisure time physical activity is associated with lower chronic disease risk in adults with spinal cord injury. Appl Physiol Nutr Metab 2009 Aug;34(4):640-7.

(30) Manns PJ, McCubbin JA, Williams DP. Fitness, inflammation, and the metabolic syndrome in men with paraplegia. Arch Phys Med Rehabil 2005 Jun;86(6):1176-81.

(31) Martin Ginis KA, Jorgensen S, Stapleton J. Exercise and sport for persons with spinal cord injury. PM and R 2012 Nov;4(11):894-900.

(32) Myers J, Kiratli BJ, Jaramillo J. The Cardiometabolic Benefits of Routine Physical Activity in Persons Living with Spinal Cord Injury. Current Cardiovascular Risk Reports 2012;6(4):323-30.

(33) Cowan RE, Nash MS. Cardiovascular disease, $\mathrm{SCl}$ and exercise: unique risks and focused countermeasures. Disabil Rehabil 2010;32(26):2228-36.

(34) Da Silva AE, De AL, V, Ruiz Da SF, Lira FS, Dos Santos RVT, Rosa JPP, et al. Low-grade inflammation and spinal cord injury: Exercise as therapy? Mediators of Inflammation 2013;2013 Article Number(971841. Date of Publication).

(35) Hamer M, Molloy GJ, de Oliveira C, Demakakos P. Leisure time physical activity, risk of depressive symptoms, and inflammatory mediators: the English Longitudinal Study of Ageing. Psychoneuroendocrinology 2009 Aug;34(7):1050-5.

(36) Howren MB, Lamkin DM, Suls J. Associations of depression with C-reactive protein, IL-1, and IL-6: a meta-analysis. Psychosom Med 2009 Feb;71(2):171-86.

(37) Aronson D, Sheikh-Ahmad M, Avizohar O, Kerner A, Sella R, Bartha P, et al. CReactive protein is inversely related to physical fitness in middle-aged subjects. Atherosclerosis 2004 Sep;176(1):173-9.

(38) Aronson D, Sella R, Sheikh-Ahmad M, Kerner A, Avizohar O, Rispler S, et al. The association between cardiorespiratory fitness and C-reactive protein in subjects with the metabolic syndrome. J Am Coll Cardiol 2004 Nov 16;44(10):2003-7.

(39) Plaisance EP, Grandjean PW. Physical activity and high-sensitivity C-reactive protein. Sports Med 2006;36(5):443-58.

(40) Steensberg A, Toft AD, Schjerling P, Halkjaer-Kristensen J, Pedersen BK. Plasma interleukin-6 during strenuous exercise: role of epinephrine. Am J Physiol Cell Physiol 2001 Sep;281(3):C1001-C1004.

(41) Febbraio MA, Pedersen BK. Muscle-derived interleukin-6: mechanisms for activation and possible biological roles. FASEB J 2002 Sep;16(11):1335-47.

(42) Gillum TL, Kuennen MR, Schneider S, Moseley P. A review of sex differences in immune function after aerobic exercise. Exerc Immunol Rev 2011;17:104-21.

(43) Pedersen BK, Febbraio M. Muscle-derived interleukin-6--a possible link between skeletal muscle, adipose tissue, liver, and brain. Brain Behav Immun 2005 Sep;19(5):371-6. 
Figures and tables

Table 1. Study characteristics on 'Effects of physical activity on inflammation in persons with 571 spinal cord injury (SCl)'.

572 Figure 1. PRISMA study flow diagram of search results for effect of physical activity on 573 circulating inflammation markers in SCl.

574 Figure 2. Cochrane risk of bias summary: review authors' judgements about each risk of bias 575 item for each included study.

576 Figure 3. Newcastle-Ottawa Scale cohort studies risk of bias summary: review authors' 577 judgements about each risk of bias item for each included study.

578 Figure 4. GRADE summary of findings of the main comparisons [Explanation].

579 Figure 5. Meta-analysis Acute IL-6 response in SCI versus able-bodied participants 580 compared to pre-exercise.

581 Figure 6. Meta analysis CRP in physically active versus physically inactive participants.

582 Figure 7. Meta analysis CRP in physically active versus physically inactive participants 583 including mode of mobility (cross-sectional).

584 Figure 8. Meta analysis Mean CRP in physically active versus physically inactive tetraplegia 585 participants (cross-sectional).

586 Figure 9 Meta analysis Mean difference in CRP level in post-training compared to pre587 training in participants with $\mathrm{SCl}$.

588 Figure 9. Meta analysis Mean difference in CRP level in post-training compared to pre589 training in participants with $\mathrm{SCl}$. 
590

591

592

593

594

595

596

597 